\title{
Nurse practitioners and interdisciplinary teams in pediatric critical care
}

\section{Authors: Kristin H. Gigli, Mary S. Dietrich, Peter I. Buerhaus, and Ann F. Minnick}

This is a postprint of an article that originally appeared in AACN Advanced Critical Care on June 2018. The final version can be found at https://dx.doi.org/10.4037/aacnacc2018588.

Gigli, Kristin H. , Mary S. Dietrich, Peter I. Buerhaus, and Ann F. Minnick. "Nurse Practitioners and Interdisciplinary Teams in Pediatric Critical Care." AACN Advanced Critical Care 29, no. 2 (June 2018): 138-148. DOI:10.4037/aacnacc2018588. 


\section{Nurse Practitioners and Interdisciplinary Teams in Pediatric Critical Care}

Kristin H. Gigli, MSN, RN

Mary S. Dietrich, PhD, MS

Peter I. Buerhaus, PhD, RN

Ann F. Minnick, PhD, RN

\section{ABSTRACT}

Objective: To describe the members of pediatric intensive care unit interdisciplinary provider teams and labor inputs, working conditions, and clinical practice of pediatric intensive care unit nurse practitioners.

Methods: A national, quantitative, crosssectional, descriptive postal survey of pediatric intensive care unit medical directors and nurse practitioners was administered to gather information about provider-team members, pediatric intensive care unit nurse practitioner labor inputs, working conditions, and clinical practice. Descriptive statistics, cross-tabulations, and $\chi^{2}$ tests were used.

Results: Responses from 97 pediatric intensive care unit medical directors and 59 pediatric intensive care unit nurse practitioners representing 126 institutions were received.
Provider-team composition varied between institutions with and without nurse practitioners. Pediatric intensive care units employed an average of 3 full-time nurse practitioners; the average nurse practitioner-to-patient ratio was 1 to 5 . The clinical practice reported by medical directors was consistent with practice reported by nurse practitioners.

Conclusion: Nurse practitioners are integrated into interdisciplinary pediatric intensive care unit teams, but institutional variation in team composition exists. Investigating models of care contributes to the understanding of how models influence positive patient and organizational outcomes and may change future role implementation.

Keywords: interdisciplinary team, pediatric intensive care unit, nurse practitioner roles

More than 2 million children are admitted to US hospitals annually. These children are hospitalized with increased illness severity and more chronic illnesses than in the past. ${ }^{2-6}$ Although a nearly $1 \%$ decrease in the number of pediatric hospitalizations was seen from 2008 to 2012, the number of admissions to the pediatric intensive care unit (PICU) increased. ${ }^{2,-9}$

Despite a significant demand for pediatric critical care delivery, a shortage of pediatric intensivists exists. ${ }^{10}$ Pediatric intensive care unit provider workforce shortages have been exacerbated by restrictions to training-physician work hours. ${ }^{10-13}$ As the role of the nurse practitioner (NP) has developed, NPs have been recognized as safe, high-quality health care 
providers. Over the past 20 years, growing numbers of NPs have been employed as hospital-based pediatric providers. ${ }^{13-19}$ Nurse practitioners in the PICU care for children who are acutely, chronically, and critically ill and adults with chronic childhood illness. ${ }^{20}$ Increasingly, PICU NPs are members of interdisciplinary care teams; however, the roles of PICU NPs in patient care delivery have not been widely evaluated..$^{21,22}$

Interdisciplinary care, teamwork, and collaboration are important components of delivering high-quality health care. ${ }^{23}$ Health care policy has focused on interdisciplinary team care as a means to improve access to care and health outcomes. ${ }^{24}$ Successful interdisciplinary teams maximize the contributions of each team member's knowledge, skills, and professional expertise. ${ }^{23}$ In critical care, interdisciplinary teams that include NPs have demonstrated improvements in patient safety, guideline adherence, and quality outcomes, along with lower patient mortality rates. ${ }^{25-29}$ However, pediatric teams have been excluded from most interdisciplinary studies. ${ }^{27}$

In the PICU, interdisciplinary teams comprise members from diverse backgrounds including medicine, nursing, pharmacy, rehabilitative therapies, nutrition, and social work. To understand how interdisciplinary PICU teams influence patient outcomes, attributes of the team and health care professionals' roles must be defined. In this article, we describe the providers (ie, PICU attending physicians; intensivists; pulmonologists; anesthesiologists; physicians in all stages of training such as critical care fellows, pediatric and nonpediatric residents, and medical students; and advanced practice providers such as NPs and physician assistants [PAs]) who serve as leaders on interdisciplinary PICU teams. These providers may have similar roles and may have been granted institutional privileges to diagnose and manage diseases or conditions and treat patients in the PICU. ${ }^{19,30}$ However, little information exists about the composition of PICU interdisciplinary provider teams and providers' roles in patient care.

The purpose of our research was to (1) describe the presence of physicians and advanced practice providers on PICU interdisciplinary provider teams; and (2) describe PICU NP labor input (ie, number of full and part-time NPs and their certifications), working conditions (ie, schedules and workloads), and clinical practice roles and responsibilities including expected procedural competency.

\section{Methods}

\section{Participants}

A national, quantitative, cross-sectional, descriptive survey of PICU medical directors and lead PICU NPs (ie, most senior NP or an NP serving in a supervisory role among a group of PICU NPs) was conducted. Institutions that reported having a PICU were identified in the 2015 American Hospital Association Annual Survey Database. ${ }^{31}$ The primary investigator placed telephone calls to each institution in the summer of 2016 to confirm the continued operation of the PICU and determine the presence of a PICU NP. If a PICU NP was employed, an attempt to identify a lead NP was made. Surveys were sent to the medical director at each operational PICU $(\mathrm{n}=326)$ and to lead PICU NPs $(\mathrm{n}=140)$.

\section{Procedures}

The development of a 34-item survey instrument was based on concepts derived from a synthesis of frameworks ${ }^{32-35}$ that examine the role of NPs in care delivery. The Vanderbilt University Medical Center's Institutional Review Board approved this study before the recruitment and distribution of study materials.

An introductory postcard and 3 separate survey mailings were sent through the US Postal Service between October 2016 and January 2017. Survey mailings included a cover letter, a definition of key concepts, a paper survey (which included an electronic participation option), and a self-addressed, stamped return envelope. Participants who returned a completed survey were eligible for a drawing of a $\$ 250$ Visa gift card (1 medical director and 1 lead PICU NP were selected as recipients).

Surveys were returned electronically $(\mathrm{n}=29$, $19 \%)$ or by mail $(\mathrm{n}=123,81 \%)$. A secure, web-based platform hosted at Vanderbilt University, Research Electronic Data Capture (REDCap), was used to capture responses. ${ }^{36}$ Electronic survey responses were entered into the REDCap system by the respondents, whereas mailed survey responses were double entered into the system by the primary investigator. Returning a survey indicated participant consent. 


\section{Variables}

Questions were included in the survey instrument that enabled us to identify the team composition of the following types of providers: pediatric attending physicians (intensivists, pulmonologists, and/or anesthesiologists), physicians in all stages of training (ie, critical care fellows, pediatric and nonpediatric residents, and medical students), and advanced practice providers (NPs and PAs). Respondents were asked to indicate the presence of a provider but not the number of providers on the interdisciplinary provider team. Respondents were asked to report the number of PICU interdisciplinary teams that work each day in the PICU.

Variables were included that described PICU NP labor inputs, working conditions, and patient care roles. Pediatric intensive care unit NP labor inputs included measures of labor quantity and descriptive attributes (A. Minnick, verbal communication, October 2014). The labor quantity variables included were the number of full-time, part-time, and per diem PICU NPs employed in the PICU; full-time equivalents were not measured. Descriptive attributes, such as the PICU NP clinical certifications as primary or acute care pediatric nurse practitioners or family nurse practitioners, were captured.

Questions assessing working conditions included work schedules, shift length, and workload. ${ }^{37}$ The routine presence or absence of PICU NPs was assessed on day and night shifts during the weekdays and on weekends. Typical patient workloads on day and night shifts, during the week, and on weekends were reported. The predominant shift length was reported for weekday and weekend day shifts. For institutions that did not have NP presence 24 hours a day, when no PICU NP was scheduled to work, the provider who was primarily responsible for the care of the NP's patients was ascertained.

Clinical practice is guided by and should be consistent with the PICU NP's education, competence, and responsibilities related to the delivery of patient care. ${ }^{38}$ For this study, the following major areas of clinical practice were assessed: clinical responsibilities including day-to-day patient care, education and training, and quality improvement leadership; requirements to maintain procedural competency; and clinical roles as respondents to acute patient-care situations within and outside of the PICU. ${ }^{15,38,39}$

\section{Data Analysis}

Data analyses were performed using SPSS, version 23.0 (IBM Corporation). Frequency distributions were used to summarize the nominal and ordinal data distributions; median and interquartile range (IQR) were used for the continuous data, because of skewness. Differences in response distributions among institutional practices and provider reports of clinical practice were analyzed using $\chi^{2}$ tests of independence $(P<.05$ was considered statistically significant).

\section{Results}

Responses were received from 97 out of 326 PICU medical directors $(30 \%)$ and 59 out of 140 lead PICU NPs (42\%), representing 126 unique institutions $(39 \%$ of all institutions with PICUs). Paired responses, 1 from the medical director and another from the lead NP, were received from 26 institutions. For institutional-level data, the physician response was included in the analyses for consistency in respondent roles.

\section{Interdisciplinary Team Composition}

Nearly $75 \%$ of respondents were PICU medical directors or lead PICU NPs from institutions that employ PICU NPs $(\mathrm{n}=93)$ (Table 1). Institutions that employed PICU NPs had larger PICUs than those that did not employ PICU NPs (a median of 20 licensed beds, with an average daily census of 14 patients vs a median of 10 licensed beds, with an average daily census of 6 patients, respectively; both $P<.001)$. Compared with institutions that employed PICU NPs, institutions that did not employ NPs were more likely to report having only 1 PICU provider team working in the PICU each day $173 \%$ vs $44 \%$ ), whereas as many as 4 PICU provider teams were reported to work daily in PICUs with NPs. The institutions without PICU NPs were also significantly less likely to have a separate pediatric cardiac intensive care unit $(15 \%$ vs $47 \%)$ or offer pediatric cardiothoracic surgery ( $23 \%$ vs $67 \%$ ) than were those institutions that employ PICU NPs $(P<.001)$ (Table 1$)$.

All respondents $(\mathrm{n}=156)$ reported that pediatric intensivists were members of the 


\section{Characteristic}

\author{
Total PICUs \\ ( $n=126$ )
}

\section{Employs PICU Nurse \\ Practitioners ( $n=93$ )}

\section{Does Not Employ PICU Nurse Practitioners ( $n=33$ )}

$10(8,12)$

$20(14,28)$

$14(10,20)$

$6(4,8)$

49 (38.9)

$34(27.0)$

$43(34.1)$ No, the institution does not offer cardiac surgery (\%)

Admission policy, ${ }^{\text {c,d }}$ No. (\%)

Open unit

Semi-open unit

Closed unit

\section{Number of ICU teams in PICU each day, No. (\%) \\ 1 \\ 2 \\ $\geq 3$}

$18(14.8)$
$34(27.9)$
$70(57.3)$

$65(51.6)$

36 (28.6)

$25(19.8)$

$44(47.3)^{a}$
$15(12,24)$
$28(30.1)^{a}$
$21(22.6)^{a}$
$17(18.5)^{a}$
$23(25.0)^{a}$
$52(56.5)^{a}$

$5(15.2)^{b}$

$16(7,18)$

$6(18.2)^{a}$

$22(66.7)^{\mathrm{b}}$

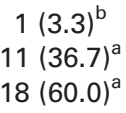

.10

.02

\footnotetext{
Abbreviations: ICU, intensive care unit; IQR, interquartile range; PICU, pediatric intensive care unit.

${ }^{a}$ b Statistically significant differences between responses from institutions that employ PICU nurse practitioners and those that do not using Bonferroni-corrected $P<.05$.

${ }^{c}$ Admission policy: $n=122$, total PICU; $n=92$, employs PICU nurse practitioners; $n=30$, does not employ PICU nurse practitioners.

${ }^{\mathrm{d}}$ Open unit: a PICU where patients receive care primarily from physicians with responsibilities outside the ICU. Critical care specialists are often available on a consultation basis. Semi-open unit: a PICU where patients receive care primarily from a physician with responsibilities outside the ICU or exclusively by critical care specialists or teams based on the nature of the illness necessitating PICU admission (eg, surgical patient). Closed unit: a PICU where patients are cared for exclusively by critical care specialists or teams.
}

PICU attending physician team; only $30 \%$ reported their teams included a pediatric pulmonologist and 19\% reported their teams included a pediatric anesthesiologist (Table 2). If an institution employed PICU NPs, it was significantly more likely to have pediatric critical care fellows, nonpediatric residents, and PAs working on its interdisciplinary PICU provider team than institutions who did not employ PICU NPs $(P<.05)$.

\section{Labor Inputs and Working Conditions}

Institutional-level responses $(\mathrm{n}=93)$ from a PICU medical director or lead PICU NP regarding PICU NP labor inputs and working conditions are shown in Table 3. Although most respondents indicated that their institutions hired PICU NPs certified as acute care pediatric NPs, only $44 \%$ of respondents reported that acute care certification was required for PICU NPs. Institutions also employed primary care-certified pediatric NPs $(26 \%)$ and family NPs (17\%); these constituted the remaining PICU NP workforce.

Respondents from institutions that employed PICU NPs indicated there was a median of 3 full-time PICU NPs (range, 0-23). Employment of part-time and per diem PICU NPs was much lower (part-time: median, 0 , range, $0-8$; per diem: median, 0 , range, $0-4$ ). When adjusted for PICU census, the median ratio of NP to patient was 1 to 4 (IQR, 1:2, 1:8). When adjusted for the number of licensed beds, the median NP to bed ratio was 1 to 5 (IQR, 1:4, 1:10).

Pediatric intensive care unit NPs were responsible for a median of 4 to 5 patients per shift (IQR, 2-8 patients with shift variation; 
Table 2: Pediatric Intensive Care Unit Interdisciplinary Provider Team Composition

\section{Team Composition}

Pediatric pulmonologists

Pediatric anesthesiologists

Pediatric critical care fellows

Pediatric residents

Nonpediatric residents

Medical students

Physician assistants

\section{Total PICUs \\ $(\mathrm{n}=124)^{\mathrm{a}}$}

37 (29.8)

$24(19.4)$

$46(37.1)$

$95(77.0)$

$68(54.8)$

$84(67.7)$

$34(27.4)$

\section{Employs PICU \\ NPs $(\mathrm{n}=91)^{\mathrm{a}}$}

27 (30)

$16(18)$

$44(48)$

$73(80)$

$55(60)$

$63(69)$

31 (34)

\section{Does Not Employ \\ PICU NPs $(n=33)^{a}$}

$\begin{array}{cc}10(30) & .75 \\ 8(24) & .83 \\ 2(6) & <.001 \\ 22(67) & .08 \\ 13(39) & .04 \\ 21(64) & .63 \\ 3(9) & .009\end{array}$

Abbreviation: PICU, pediatric intensive care unit.

${ }^{\mathrm{a}}$ Number, (\%)

Table 3: Characteristics of Labor Input and Working Conditions for Nurse Practitioners in Pediatric Intensive Care Units ${ }^{a}$

\section{Characteristic}

NP certification, No. (\%)

Pediatric nurse practitioner-acute care

Require acute care certification

Pediatric nurse practitioner-primary care

Family nurse practitioner

No. of NPs, median (IOR) (range)

Full time

Part time

Per diem
Employs PICU NPs

( $n=93$ )

$84(90)$

$41(44)$

$24(26)$

$16(17)$

$3(1,5)(0-23)$

$0(0,1)(0-8)$

$0(0,0)(0-4)$

$5(4,8)$

$4.25(0,12.5)$

$5(3.5,10)$

$5(0,12)$

$22(24)$

67 (72)

26 (28)

47 (51)

$13(14)$

$12(10,12)(8-24)$

$12(8,12)(3-24)$

Typical shift length, median (IOR) (range)

Week day

Weekend day 
Deliver patient care

Educate nurse practitioners

Lead quality improvement

Train physicians

Educate physicians

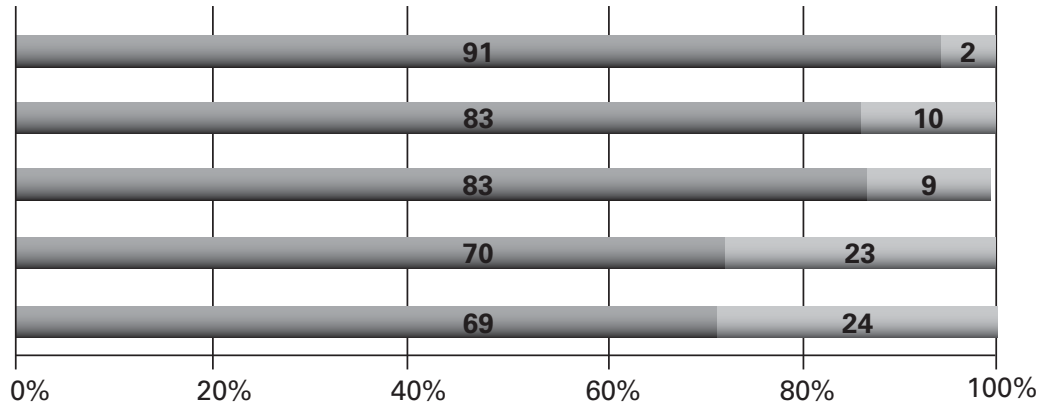

$0 \%$

$20 \%$

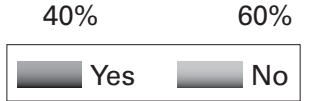

Figure 1: Clinical responsibilities of pediatric intensive care unit nurse practitioners $(n=93)$. Numbers in the bars represent the number of respondents who answered.

range, 1-20 patients). If an institution did not have PICU NP presence 24 hours a day, the PICU attending physicians most frequently assumed management responsibility for PICU NPs' patients when no NP was working.

In one-quarter of institutions, respondents reported PICU NPs provide care 24 hours a day. If NP staffing is allowed, a few PICUs reported increasing their PICU NP coverage to 24 hours a day. In those institutions that did not have PICU NP presence 24 hours a day, most respondents $(72 \%)$ reported that a PICU NP is present on day shifts during the week and 50\% reported PICU NPs are present during day shifts on the weekend. Two institutions employed PICU NPs to provide overnight coverage only. The median PICU NP shift length was 12 hours, with almost one-third of institutions operating 24-hour NP shifts.

\section{Nurse Practitioner Clinical Practice}

Expectations of medical directors who worked at institutions employing PICU NPs $(\mathrm{n}=68)$ and lead NPs $(\mathrm{n}=59)$ were compared. No statistically significant differences in expectations of PICU NPs' clinical responsibilities, need for procedural competency, or roles were identified between PICU medical directors and lead NPs; therefore, overall responses to the survey are described by institution.

Virtually all respondents reported PICU NPs participated in the delivery of direct patient care. Most PICU NPs participate in educating NPs and have a role in the training and education of physicians (Figure 1). Most respondents reported institutional requirements for procedural competency among PICU NPs as part of the provision of day-to-day patient care. Lumbar puncture was reported as a required competency most often $(90 \%)$ and chest tube insertion was reported least often (68\%) (Figure 2). A higher percentage of lead PICU NPs consistently reported that NPs were required to have procedural competencies in the areas shown than did the group of medial directors (Figure 3).

The roles of PICU NPs as team members who respond to acute patient care situations within and outside of the PICU are summarized in Figure 4. The most commonly reported roles NPs were expected to play in responding to acute patient situations were as members of the code and rapid response teams. However, PICU NPs were reported to lead those teams less than half of the time. Rarely were PICU NPs required to respond to a trauma stat situation.

\section{Discussion}

In this national study, we examined PICU interdisciplinary care teams, PICU NP labor input, working conditions, and clinical practice roles and responsibilities on the care team. This is a first step toward evaluating the care delivery of an interdisciplinary PICU provider workforce. As PICU NPs are integrated into PICU provider care teams, knowledge of their role has implications for standardization of NP integration and use on PICU care teams, NP educational preparation, and assessing NPs' influence on patient outcomes.

\section{Interdisciplinary Teams}

Institutions that reported employing PICU NPs had more licensed beds and a higher 
Perform lumbar puncture

Arterial line placement

Central venous line placement

Chest tube removal

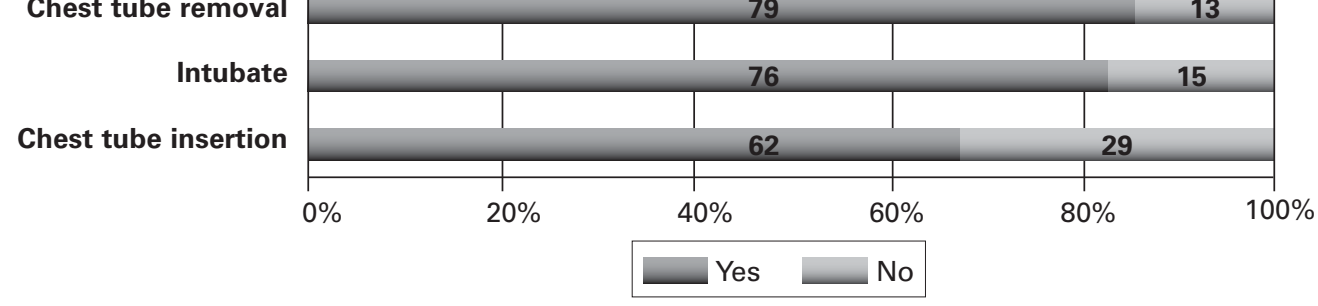

Figure 2: Procedural competencies expected of pediatric intensive care unit nurse practitioners as part of clinical practice in the United States $(n=93)$. Numbers in the bars represent the number of respondents who answered yes or no.

Perform lumbar puncture

Arterial line placement

Central venous line placement

Chest tube removal

Intubate

Chest tube insertion

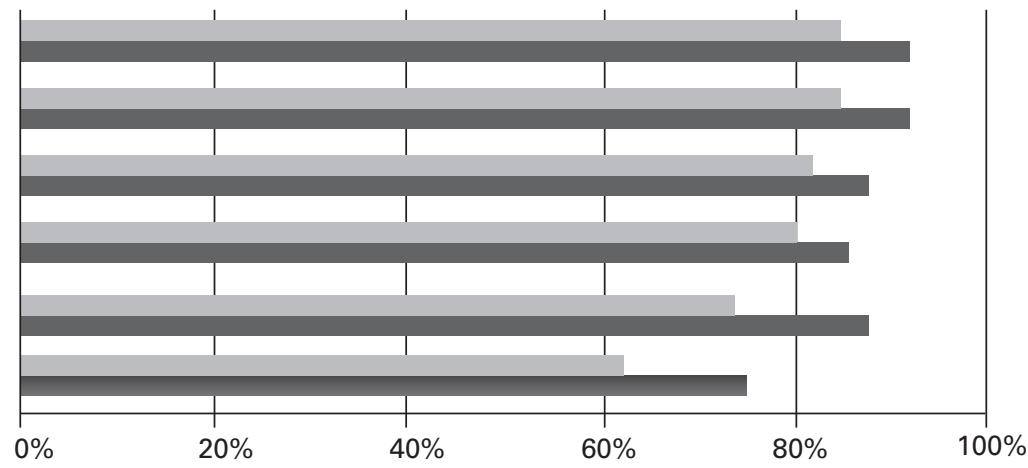

Reported by PICU medical directors

Reported by lead PICU NPs

Figure 3: Differences in expectations of pediatric intensive care unit nurse practitioners' procedural competencies as reported by medical directors $(n=68)$ and lead PICU NPs $(n=59)$.

Abbreviations: NP, nurse practitioner; PICU, pediatric intensive care unit.

\section{Code team member}

Rapid response team member

\section{Consultant to medical patients outside the ICU}

Consultant to surgical patients outside ICU

Rapid response team leader

Code team leader

Trauma stat responder

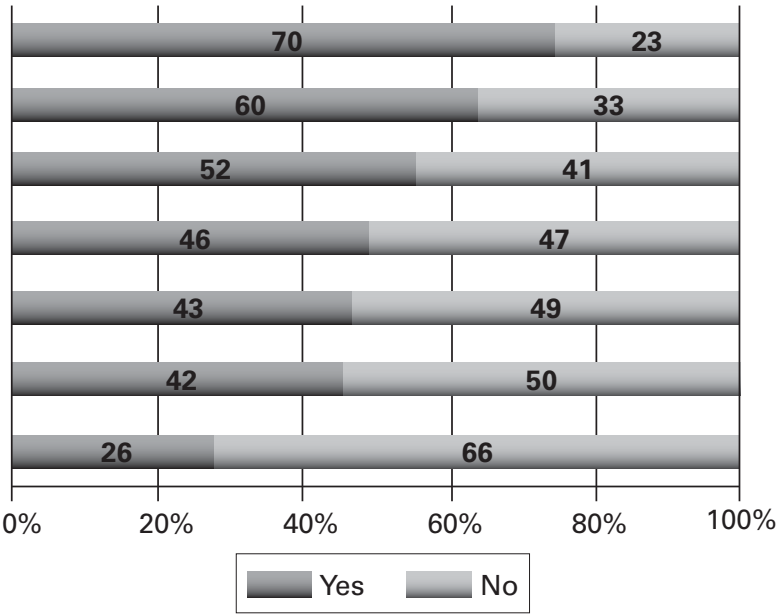

Figure 4: Clinical roles in acute patient care situations as part of clinical practice of pediatric intensive care unit nurse practitioners ( $n=93$ ). Numbers in the bars represent the number of respondents who answered yes or no. Abbreviation: ICU, intensive care unit. 
average daily census in the PICU than those who reported not employing PICU NPs. In larger PICUs, more patients require more providers and, as seen in adult intensive care units, the PICU NP's presence can be part of an innovative model of care delivery. ${ }^{40}$ The institutions that employ PICU NPs are significantly more likely to have a separate pediatric cardiac ICU. Assessment of the role of pediatric cardiac ICU NPs has not been reported and should be considered in future studies of the NP role in the delivery of care to children in critical care settings.

Institutional variation exists in the composition of the interdisciplinary PICU provider team. Compared with adult intensive care-provider teams, interdisciplinary PICU teams report fewer attending pulmonologists and anesthesiologists in direct PICU patient care. ${ }^{10}$ In adult critical care, they make up a significant portion of the workforce and, given the relatively recent addition of pediatric critical care medicine as a specialty, the fact that there are fewer pulmonologists and anesthesiologists in the PICU is an important finding for forecasts of the PICU physician workforce.

More respondents reported critical care fellowship programs at institutions that employ PICU NPs than at institutions without PICU NPs. These institutions may be those in academic medical centers, which are more likely to have medical and nursing schools and thus have more critical care fellows and NP representation on the interdisciplinary team. ${ }^{41}$ The relationships between critical care fellows and PICU NPs could shape future interdisciplinary collaboration, because fellows go on to work in positions as attending PICU physicians. Evaluation of the relationships between these providers as they interact in training, education, and patient-care delivery should be a focus of future studies. ${ }^{42}$

Consistent with findings of prior PICU workforce studies, ${ }^{19,26}$ we found the presence of PAs on PICU interdisciplinary teams is less common than that of PICU NPs. Although reasons for the smaller presence of PAs on PICU interdisciplinary teams is unknown, greater use of PAs may be an alternative way to grow the PICU workforce. Developing knowledge of the PA roles and experiences in PICU care relative to NPs would be a useful area for research.
Other interdisciplinary team members, including bedside nurses, respiratory therapists, and pharmacists, among others, were not included in this study, even though they contribute to positive patient outcomes in the PICU. ${ }^{43}$ Future studies of these health care professionals' roles and their potential influence on PICU physician, NP, and PA roles are needed to understand more comprehensively PICU team dynamics.

\section{Labor Inputs and Working Conditions}

Although most respondents reported that PICU NPs are acute care pediatric NPs, not all PICU NPs reported they were acute care pediatric NPs; fewer than one-half of institutions require PICU NPs to have acute care certification. Efforts to align NP education, certification, licensure, and practice are needed to encourage the use of acute care pediatric NPs in the PICU. ${ }^{44,45}$ The expansion of graduate-level opportunities allowing for acute care pediatric NP education may support the demand for more PICU NPs. ${ }^{46}$ Employers who hire new PICU NPs to join their interdisciplinary teams should be aware that practice in a PICU is considered acute and critical care, and requires acute careeducated and certified pediatric NPs. ${ }^{15}$

A dose of NP has been described to determine how NP providers influenced chronic management of pediatric patient care. ${ }^{47}$ The dose measure includes labor inputs and working conditions. We describe in this study the factors that contribute to a dose of NP care in a novel setting, the PICU. Findings related to labor inputs and working conditions may be useful for future studies assessing the dose of PICU NP care, as similar to chronic care management, that PICU NPs typically contribute as part of an interdisciplinary provider team. Similarly, studies are needed to assess the adequacy of the available PICU NP workforce to meet the dose demands and determine future workforce development needs. Efforts to associate patient and organizational outcomes with the presence of PICU NPs will require intentional assessment of the dose of NP, particularly because most PICUs do not have a continuous NP-coverage model.

A description of other non-NP provider member roles and contributions to patient care should be examined; these are important to understand when considering patient 
outcomes. Ultimately, efforts to integrate PICU NPs into the interdisciplinary provider team should seek to optimize the contributions of all team members' clinical skills, knowledge, roles, and responsibilities to optimize the workforce and pediatric outcomes.

\section{Nurse Practitioner Clinical Practice}

The clinical practice expectations reported for PICU NPs were similar to findings in a prior study. ${ }^{21}$ The PICU NP is primarily responsible for the day-to-day provision of direct patient care. Development of more specific knowledge of NP roles associated with the day-to-day care of PICU patients, including, for example, ventilator management, titration of vasoactive medications, and care coordination, and professional roles in research, leadership, and education, will allow for PICU NP clinical practice patterns and variations in practice to be examined. ${ }^{21}$

Trends in professional role opportunities for PICU NPs should be monitored because provider shortages may necessitate more clinical time dedicated to the provision of dayto-day patient care. Examination of PICU NPs' participation in professional roles and the implications for PICU NP role actualization, role satisfaction, and retention may support the clinically focused PICU NP to have opportunities to engage in professional development activities.

Patient-to-NP ratios also should be further evaluated in focused studies of NP roles. Factors including expectations of the PICU NP role in care delivery, patient acuity, providerteam composition, and patient census may contribute to the wide range in patient-to-NP ratios reported in this study. ${ }^{48}$ Given a wide range of reported ratios, no conclusions about optimal patient-to-NP ratio can be drawn from this study.

Medical director-reported and lead PICU NP-reported expectations for PICU NP roles in the response to acute patient care situations (eg, codes, rapid response, consultations outside of the PICU) showed less standardized practice expectations for NPs across respondent institutions. Reasons for variations in practice and expectations were not explored in this study. However, institutional culture and climate, including a unit's values and working conditions, should be considered. ${ }^{49}$ Qualitative studies can contribute to the knowledge of factors associated with variations in PICU NP practice reported among institutions.

To our knowledge, this is the first study in which medical director and lead PICU NP expectations for PICU NPs' clinical practice were compared. Although not statistically significant, discrepancy in reports of expectations for procedural competency between the medical directors and lead PICU NPs and variation in PICU NP role expectations were noted between institutions. Studies in which variation in PICU NP clinical practice are evaluated may elicit physicians' perceptions of the value PICU NPs bring to patient care. Physicians in the PICU report a desire to hire additional PICU NPs and expand the role of those providers. ${ }^{11}$ Studies of the aspects of the NP role they would like to expand are necessary and have implications for PICU NP workforce planning and education.

\section{Considerations for Future Work}

We focused on the clinical practice of the PICU NP. Research on the detailed care PICU NPs provide, including specific patient management and treatment roles along with professional role expectations, would build knowledge of the contributions of PICU NPs to patient and organizational outcomes. Comparison studies of similarities and differences between PICU NP and other hospital-based, acute care pediatric NP roles are needed. These studies have potential implications for the education, training, and institutional orientation for all hospital-based, pediatric NPs.

Our analysis was limited to examining NP clinical practice only in institutions that currently employ PICU NPs. We suggest the expectations for NP practice among PICU medical directors from institutions that do not currently employ PICU NPs but plan to have NPs join their interdisciplinary provider team could be examined in future studies. Comparisons of the expectations of medical directors who desire to work with PICU NPs and those who currently work with PICU NPs may highlight the value physicians view NP team members bring to patient care. Such findings may have implications for role development of PICU NPs.

\section{Limitations}

In describing the interdisciplinary teams, this study is limited by reporting the presence or absence of a provider on the PICU 
interdisciplinary team. More meaningful descriptions of the interdisciplinary providerteam composition could have been created through more detailed reports of labor input and working conditions for each member of the provider team. In addition, understanding practice expectations for all team members would support additional comparisons of other PICU providers' clinical practice expectations with those of the PICU NP.

Findings regarding PICU NP roles and responsibilities and patient-to-NP ratios are limited by self-report rather than observation, which may lead to inaccuracies. Only the major clinical roles of the PICU NP were examined in this study, not specific management responsibilities for patient care; as a result, factors that influence institutional expectations of the ratio of PICU provider to patient could not be determined from this study. In addition, institutional culture and individual provider characteristics (eg, level of experience), both of which may contribute to a PICU NP's role and integration into an interdisciplinary PICU care team, were not accounted for in this study.

\section{Conclusion}

Our intent in this study was to describe the presence of physician and advanced practice provider members on PICU interdisciplinary care teams, as well as PICU NP labor input, working conditions, and clinical practice roles and responsibilities on the care team. Interdisciplinary PICU provider teams will become more common as the PICU physician shortage continues. Pediatric ICU NPs are members of interdisciplinary PICU teams and are active in providing patient care and educating team members. Assessing the dose of NP on the interdisciplinary care team will support measurement of PICU NPs' contribution to patient and organizational outcomes. Applying PICU NP labor inputs and working conditions to future inquiry of other interdisciplinary care team members can clarify how provider teams function to deliver care to patients in the PICU.

The clinical practice of PICU NPs is consistent with that reported in a prior study of PICU NP roles. ${ }^{21}$ In the PICU, NPs have roles in contributing to patient care. Detailed descriptions of the aspects of patient care and professional role expectations of PICU NPs will enable comparisons of the role with that of other pediatric hospital-based NPs and build understanding of the unique aspects of the PICU NP role. Determining the aspects of the PICU NP practice that are most valuable to physicians and have a positive influence on patient-care outcomes may change the way the role is implemented in the future; thus, these aspects deserve consideration. With an interdisciplinary care team, the team composition and all providers' practices should be further evaluated to assess how models of care contribute to patient care outcomes. With ongoing examination and future interventional studies, research can assist in determining how to optimize the integration of PICU NPs into the interdisciplinary PICU provider teams.

\section{ACKNOWLEDGMENT}

We thank Ruth Kleinpell, PhD, RN, FAAN, FCCM, for thoughtful review of the manuscript.

\section{REFERENCES}

1. Agency for Healthcare Research and Quality. HCUP Kids' Inpatient Database (KID) website. http://www.hcup-us .ahrq.gov/kidoverview.jsp. Accessed May 15, 2017.

2. Witt WP, Weiss AJ, Elixhauser A. Overview of hospital stays for children in the United States, 2012. HCUP Statistical Brief \#187. Rockville, MD: Agency for Healthcare Research and Quality; 2014. http:// www.hcup-us.ahrq.gov/reports/statbriefs/sb187 -Hospital-Stays-Children-2012.pdf. Published December 2014. Accessed March 27, 2018.

3. Edwards JD, Houtrow AJ, Vasilevskis EE, et al. Chronic conditions among children admitted to U.S. pediatric intensive care units: their prevalence and impact on risk for mortality and prolonged length of stay. Crit Care Med. 2012;40(7):2196-2203.

4. Hartman ME, Linde-Zwirble WT, Angus DC, Watson RS. Trends in the epidemiology of pediatric severe sepsis. Ped Crit Care Med. 2013;14(7):686-693.

5. Hasegawa K, Tsugawa Y, Brown DF, Mansbach JM, Camargo CA Jr. Trends in bronchiolitis hospitalizations in the United States, 2000-2009. Pediatrics. 2013;132(1):28-36.

6. Miller RL, Gebremariam A, Odetola FO. Pediatric highimpact conditions in the United States: retrospective analysis of hospitalizations and associated resource use. BMC Pediatr. 2012;12:61. doi:10.1186/1471-2431-12-61.

7. Watson RS, Hartman ME. Epidemiology of critical illness. In: Wheeler DS, Wong HR, Shanley TP, eds. Pediatric Critical Care Medicine. Volume 1: Care of the Critically III or Injured Child. 2nd ed. London, England: SpringerVerlag; 2014.

8. Goh AY, Mok Q. Centralization of paediatric intensive care: are critically ill children appropriately referred to a regional center? Intensive Care Med. 2001;27(4):730-735.

9. Randolph AG, Gonzales CA, Cortellini L, Yeh TS. Growth of pediatric intensive care units in the United States from 1995 to 2001. J Pediatr. 2004;144(6):792-798.

10. Duke EM; US Department of Health and Human Services; Health Resources and Services Administration. Report to Congress: The critical care workforce: a study of the supply and demand for critical care physicians. http://www.mc.vanderbilt.edu/documents/CAPNAH /files/criticalcare.pdf. Published May 2006. Accessed May 5, 2017. 
11. Freed GL, Dunham KM, Loveland-Cherry C, et al. Nurse practitioners and physician assistants employed by general and subspecialty pediatricians. Pediatrics. 2011; 128(4):665-672.

12. Accreditation Council for Graduate Medical Education. History of duty hours. http://www.acgme.org/What-We -Do/Accreditation/Clinical-Experience-and-Education -formerly-Duty-Hours/History-of-Duty-Hours. Accessed May 15, 2017.

13. Freed GL, Dunham KM, Moran LM, Spera L. Resident work hour changes in children's hospitals: impact on staffing patterns and workforce needs. Pediatrics. 2012; 130(4):700-704.

14. Martin SA. The pediatric critical care nurse practitioner evolution and impact. Pediatr Nurs.1999;25(5):505-510.

15. Sorce L, Simone S, Madden M. Educational preparation and postgraduate training curriculum for pediatric critical care nurse practitioners. Ped Crit Care Med. 2010; 11(2):205-212.

16. Dill MJ, Pankow S, Erikson C, Shipman S. Survey shows consumers open to a greater role for physician assistants and nurse practitioners. Health Aff (Millwood). 2013; 32(6):1135-1142.

17. Kuo YF, Loresto FL Jr, Rounds LR, Goodwin JS. States with the least restrictive regulations experienced the largest increase in patients seen by nurse practitioners. Health Aff (Millwood). 2013;32(7):1236-1243.

18. Newhouse RP, Stanik-Hutt J, White KM, et al. Advanced practice nurse outcomes 1990-2008: a systematic review. Nurs Econ. 2011;29(5):230-250.

19. Freed GL, Dunham KM, Loveland-Cherry C, Martyn KK, Moote MJ. Nurse practitioners and physician assistants employed by general and subspecialty pediatricians. Pediatrics. 2011;128(4):665-672.

20. American Nurses Association; National Association of Pediatric Nurse Practitioners; Society of Pediatric Nurses. Pediatric Nursing: Scope and Standards of Practice. 2nd ed. Silver Spring, MD: American Nurses Association; 2015.

21. Verger JT, Marcoux KK, Madden MA, Bojko T, Barnsteiner $\mathrm{JH}$. Nurse practitioners in pediatric critical care: results of a national survey. AACN Clin Issues. 2005;16(3):396-408.

22. Brown AM, Christie LM, Higgerson RA, et al. A nationa survey of PICU nurse practitioners-opportunities to standardize practices and optimize service delivery. Pediatr Nurs. 2012;38(5):249-250,277.

23. Institute of Medicine of the National Academies. The Future of Nursing: Leading Change, Advancing Health. Washington, DC: The National Academies Press; 2011. http://books.nap.edu/openbook.php?record_id=12956 \&page=R1. Accessed May 18, 2017.

24. Interprofessional Education Collaborative Expert Panel. Core Competencies for Interprofessional Collaborative Practice: Report of an Expert Panel. Washington, DC: Interprofessional Education Collaborative; 2011.

25. Gershengorn HB, Wunsch H, Wahab R, et al. Impact of nonphysician staffing on outcomes in a medical ICU. Chest. 2011;139(6):1347-1353.

26. Moote M, Krsek C, Kleinpell R, Todd B. Physician assistant and nurse practitioner utilization in academic medical centers. Am J Med Qual. 2011;26(6):452-460.

27. Kim MM, Barnato AE, Angus DC, Fleisher LA, Kahn JM The effect of multidisciplinary care teams on intensive care unit mortality. Arch Intern Med. 2010;170(4):369-376.

28. Yoo EJ, Edwards JD, Dean ML, Dudley RA. Multidisciplinary critical care and intensivist staffing: results of a statewide survey and association with mortality. J Inten sive Care Med. 2016;31(5):325-332.

29. Ward NS, Afessa B, Kleinpell R, et al. Intensivist/patient ratios in closed ICUs: a statement from the Society of Critical Care Medicine Taskforce on ICU Staffing. Crit Care Med. 2013;41(2):638-645.

30. The Joint Commission. Ambulatory care program: the who, what, when and where's of credentialing and privileging. http://www.jointcommission.org/assets/1 /6/AHC_who_what_when_and_where_credentialing _booklet.pdf. Accessed May 18, 2017.

31. American Hospital Association. AHA Annual Survey Database. https://www.ahadataviewer.com/additional -data-products/AHA-Survey/ Accessed May 18, 2017.

32. Lomas J. Connecting research and policy. ISUMA Can J Policy Res. 2000(1);140-144.

33. Feldstein PJ. Health Care Economics. 6th ed. Boston, MA Delmar-Cengage Learning; 2004:436-458.

34. Lubell M, Scholz J, Berardo R, Robins G. Testing policy theory with statistical models of networks. Policy Stud J. 2012;40(3):351-374.

35. Kilpatrick K, Lavoie-Tremblay M, Lamothe L, Ritchie JA, Doran D. Conceptual framework of acute care nurse practitioner role enactment, boundary work, and perceptions of team effectiveness. J Adv Nurs. 2013;69(1):205-217.

36. Harris PA, Taylor R, Thielke R, et al. Research electronic data capture (REDCap) - a metadata-driven methodology and workflow process for providing translational research informatics support. J Biomed Inform. 2009;42(2):377-381

37. Garland A, Gershengorn HB. Staffing in ICUs: physicians and alternative staffing models. Chest. 2013;143(1):214-221.

38. National Organization of Nurse Practitioner Faculties. Population-focused nurse practitioner competencies. http://c.ymcdn.com/sites/www.nonpf.org/resource /resmgr/Competencies/CompilationPopFocusComps 2013.pdf. Published 2013. Accessed May 5, 2017.

39. Reuter-Rice K. Acute care pediatric nurse practitioner: a practice analysis study. J Pediatr Health Care. 2013; 27(6):410-418.

40. Kahn JM, Rubenfeld GD. The myth of the workforce crisis. Why the United States does not need more intensivist physicians. Am J Respir Crit Care Med. 2015;191(2):128-134

41. Accreditation Council for Graduate Medical Education. ACGME program requirements for graduate medical education in pediatric critical care medicine. https://www .acgme.org/Portals/O/PFAssets/ProgramRequirements /323_pediatric_critical_care_medicine_2017-07-01.pdf ?ver=2017-06-30-083428-177. Revised September 30, 2012. Effective July 1, 2013. Accessed June 4, 2017.

42. Hobgood C, Sherwood G, Frush K, et al; Interprofessional Patient Safety Education Collaborative. Teamwork training with nursing and medical students: does the method matter? Results of an interinstitutional, interdisciplinary collaboration. Qual Saf Health Care. 2010;19(6):e25.

43. Wang T, Benedict N, Olsen KM, et al. Effect of critical care pharmacist's intervention on medication errors: a systematic review and meta-analysis of observational studies. J Crit Care. 2015;30(5):1101-1106.

44. Bolick BN, Bevacqua J, Kline-Tilford A, et al. Recommendations for matching pediatric nurse practitioner education and certification to pediatric acute care populations. $J$ Pediatr Health Care. 2013;27(1):71-77.

45. Haut C, Madden M. Hiring appropriate providers for different populations: acute care nurse practitioners. Crit Care Nurse. 2015;35(3):e1-e8.

46. Freed GL, Moran LM, Dunham KM, Hawkins-Walsh E, Martyn KK. Capacity of, and demand for, pediatric nurse practitioner educational programs: a missing piece of the workforce puzzle. J Prof Nurs. 2015;31(4):311-317.

47. Looman WS, Presler E, Erickson MM, et al. Care coordination for children with complex special health care needs: the value of the advanced practice nurse's enhanced scope of knowledge and practice. J Pediatr Health Care. 2013;27(4):293-303.

48. Kleinpell R, Ward NS, Kelso LA, Mollenkopf FP, Hough ton $D$. Provider to patient ratios for nurse practitioners and physician assistants in critical care units. Am J Crit Care. 2015;24(3):e16-e21.

49. Poghosyan P, Nannini A, Stone PW, Smaldone A. Nurse practitioner organizational climate in primary care settings: implications for professional practice. J Prof Nurse. 2013:29(6):338-349. 